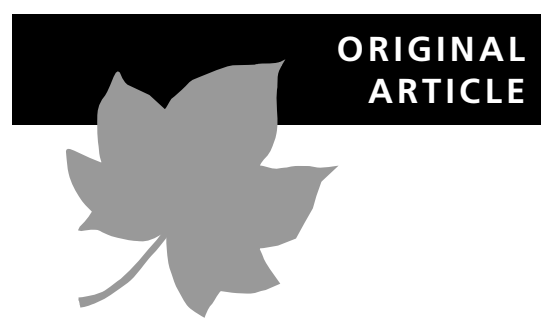

\title{
Contrasting phylogeographical patterns of two closely related species, Machilus thunbergii and Machilus kusanoi (Lauraceae), in Taiwan
}

\author{
Su-Hwa $\mathrm{Wu}^{1 \dagger}$, Ching-Yuan Hwang ${ }^{1 \dagger}$, Tsan-Piao Lin ${ }^{2}$, Jeng-Der Chung ${ }^{3}$, \\ Yu-Pin Cheng ${ }^{4}$ and Shih-Ying Hwang ${ }^{1 \star}$
}

\footnotetext{
${ }^{1}$ Graduate Institute of Biotechnology, Chinese Culture University, Yangmingshan, Taipei,

${ }^{2}$ Institute of Plant Biology, National Taiwan

University, Taipei and Divisions of

${ }^{3}$ Silviculture and ${ }^{4}$ Forest Biology, Taiwan

Forestry Research Institute, Taipei, Taiwan
}

\begin{abstract}
Aim The purpose of this paper was to study the patterns of genetic variation, demographic history, haplotype relationships and potential location of diversity centres of two closely related species, Machilus thunbergii and Machilus kusanoi.

Location The phylogeography of $M$. thunbergii and M. kusanoi was examined by sampling 110 and 106 individuals from 25 and 16 sampling sites, respectively, across their distributional range in Taiwan. Machilus thunbergii is distributed on the Asian mainland, South Korea, southern Japan, the Ryukyus, Taiwan and the Philippines, whereas M. kusanoi is endemic to Taiwan. These two species are closely related, and both are widely distributed in Taiwan but occupy different altitudinal zones and habitats.
\end{abstract}

Methods The range-wide variation of $M$. thunbergii and M. kusanoi in Taiwan was studied using chloroplast DNA (cpDNA) variations. A haplotype network was constructed with the computer program TCs. Nested clade analysis was conducted with the computer program GeoDis, and various parameters of genetic diversity were calculated and neutrality tested by the computer program DNASP. Population differentiation was estimated using the programs ARLEQUIN and HAPSTEP. The contribution of the populations to gene diversity and to allelic richness was calculated using the software CONTRIB. The level of divergence for each population from the remaining populations was calculated as the mean value of pairwise $F_{\mathrm{ST}}$ for each population against the rest of the populations.

Results Extremely low levels of genetic differentiation were found for both species. This result suggested that these two species probably survived in multiple relict refugia with different population sizes throughout the island during lowtemperature periods of the Pleistocene. In addition, nested clade analysis (NCA) of cpDNA haplotypes indicated that restricted gene flow with isolation-bydistance characterized the recolonization after the Pleistocene by Tashueshan and Shiouhluan populations of $M$. thunbergii in the north-central area west of the Central Mountain Range (CMR). In contrast, NCA analysis indicated that a major diversity centre on the southern tip of the island (Kending population) and contiguous range expansion characterized the recolonization by M. kusanoi of northern areas along the east side of the CMR. The major diversity centres found for the two species examined were further supported by the results of the mean $F_{\mathrm{ST}}$ for individual populations in comparison with other populations, and of the contribution of the divergence component to the total diversity.

Main conclusions This research supports the multiple relict refugia hypothesis for both species investigated. Populations of $M$. thunbergii at Shiouhluan and Tashueshan in the north-central area west of the CMR represent a diversity centre currently expanding its size. A diversity centre at the southern-edge population of
${ }^{\star}$ Correspondence: Shih-Ying Hwang, Graduate Institute of Biotechnology, Chinese Culture University, 55 Hwagan Road, Yangmingshan, Taipei, Taiwan 11114.

E-mail: hsy9347@ms34.hinet.net

$\dagger$ The first two authors contributed equally to this work. 
M. kusanoi, and a contiguous range expansion from Kending, were found. These results indicate that the M. thunbergii populations at Tashueshan and Shiouhluan and the M. kusanoi population at Kending, and even Soukar, are evolutionarily significant units for conservation programmes.

\section{Keywords}

Conservation, cpDNA, diversity centre, Machilus kusanoi, Machilus thunbergii, phylogeography, Taiwan.

\section{INTRODUCTION}

Interpretations on the historical framework of the geographical distribution of genetic variation in a species require an understanding of the spatial and temporal patterns that underlie population processes. Early studies used intuitive approaches to decipher phylogeographical data (Avise, 2000). Nested clade analysis (NCA) is a powerful tool for examining the geographical associations of haplotypes within a statistical framework. Knowles \& Maddison (2002) raised concerns that NCA may lead to erroneous conclusions, and suggested that both intuitive and NCA approaches should be used in phylogeographical studies. Subsequently, NCA was examined on 150 actual data sets and performed well overall, but with some false positives (Templeton, 2004). Therefore the NCA inference key was modified to reduce the incidence of false positives. NCA allows for the analysis of more complex population processes, and provides a more objective assessment of the geographical partitioning of haplotypes.

Taiwan is an island supporting tropical to cool climate vegetation, due to its low latitude and high elevations approaching $4000 \mathrm{~m}$. The Central Mountain Range (CMR) extends north to south following the axis of the island, and contains over 200 peaks $>3000 \mathrm{~m}$ in elevation. The CMR is generally divided into western and eastern parts, with the western part divided into the Shueshan Range in the north and the Yushan Massif in the south. The CMR forms the backbone of the eastern part. Although an individual peak may reach an elevation of more than $3000 \mathrm{~m}$, the relief between two peaks is rarely more than $200 \mathrm{~m}$. The remnants of glaciation at the top of some peaks indicate the former occurrence of glaciers (Böse, 2004). Ice coverage in the CMR is assumed to have been incomplete, allowing for forest patches to persist as refugia for temperate to subtropical species. This constitutes the multiple refugia hypothesis for taxa distributed today across latitudinal temperate to subtropical zones, as opposed to a single-refugium scenario. NCA analysis is probably suitable for revealing population processes for diverse groups of plants that are distributed in different altitudinal zones.

Recolonization patterns of many vascular plants after the Pleistocene can be examined by investigating representative tree species from different elevations. Moreover, historically unique populations or lineages can be identified for conser- vation efforts. Chloroplast DNA (cpDNA) is inherited maternally through seeds alone in most angiosperms, so phylogeographical analysis of cpDNA haplotypes can provide insights into recolonization and seed dispersal (Ennos, 1994). Phylogeographical studies based on cpDNA variations for widely distributed plants in Taiwan have been reported for Cyclobalanopsis glauca (Huang et al., 2002); Cunninghamia konishii (Hwang et al., 2003); and Trochodendron aralioides (Huang et al., 2004). Cyclobalanopsis glauca is a subtropicaltemperate species, whereas C. konishii and T. aralioides are both temperate species. Potential diversity centres located in the Shueshan Range, which is in the north-central area west of the CMR, were found consistently for these two temperate species. However, one diversity centre was inferred in the southern part of Taiwan for C. glauca (Huang et al., 2002), which is different from the two temperate species C. konishii and $T$. aralioides. It is thus interesting to investigate whether another species distributed in both subtropical and temperate altitudes ( $M$. thunbergii) shares a common diversity centre with C. glauca. Furthermore, the similarity and discrepancy in genetic diversity, phylogeographical structuring, and recolonization and dispersal patterns between the two closely related species $M$. thunbergii and $M$. kusanoi are worth investigating.

Machilus (Lauraceae) are evergreen trees or shrubs which consist of about 100 species distributed mainly in the tropical and subtropical areas of Asia (Liu et al., 1994). Machilus thunbergii Sieb. \& Zucc. is a large, evergreen tree distributed widely in the Asian continent, southern Korea, Japan, the Bonin islands, the Ryukyus, Taiwan and the Philippines. Machilus kusanoi (Hay.) [=Machilus japonica Sieb. \& Zucc. var. kusanoi (Hay.) Liao] is endemic to Taiwan and is distributed from the lowlands to $1400 \mathrm{~m}$, but occurs mainly within the Ficus-Machilus Zone $\left(<500 \mathrm{~m}, \quad 23-26{ }^{\circ} \mathrm{C}\right)$ ( $\mathrm{Su}, 1984)$. Machilus thunbergii is distributed at about 200 $2000 \mathrm{~m}$ and occurs mainly in the Machilus-Castanopsis zone (500-1500 m, 17-23 $\left.{ }^{\circ} \mathrm{C}\right)(\mathrm{Su}, 1984)$.

We investigated whether M. thunbergii and M. kusanoi, two closely related species with different altitudinal distributions, differ in their patterns of genetic diversity and recolonization dispersal. We also tested whether the existence of a single refugium or multiple refugia most adequately explains the phylogeographical patterns of these two closely related species. To answer these questions, we 
analysed variation in two chloroplast intergenic spacers within a statistical phylogeographical framework using coalescent mean $F_{\mathrm{ST}}$ for individual populations in comparison with the rest and divergence component contributions to the total diversity, and nested clade analysis to test hypotheses about population history.

\section{MATERIALS AND METHODS}

\section{Plant materials and DNA purification}

A total of 110 and 106 individuals were randomly sampled from 25 and 16 populations in Taiwan, encompassing the distributional range of $M$. thunbergii and $M$. kusanoi, respectively. Four individuals of $M$. thunbergii from Okinawa (the Ryukyus) were also collected. The population code, sample size, longitude and latitude for each population within Taiwan are shown in Table 1; collection sites are depicted in Fig. 1. Total DNA was extracted from ground leaf powder according to a modified cetyltrimethyl ammonium bromide (CTAB) procedure (Doyle \& Doyle, 1987), described in detail by Hwang et al. (2001). DNA was precipitated with ethanol, and after washing with $70 \%$ ethanol was dissolved in $200 \mu \mathrm{L}$ TE buffer ( $\mathrm{pH}$ 8.0) and stored at $-20{ }^{\circ} \mathrm{C}$. The DNA concentration was determined for each sample using GeneQuant II RNA/DNA Calculator (Amersham Biosciences, Taipei, Taiwan).

\section{Primers, PCR amplification and sequencing}

Polymerase chain reactions (PCR) were performed with universal primers for $\operatorname{trn} \mathrm{V}-\operatorname{trn} \mathrm{M}$ and $\operatorname{trn} \mathrm{L}-\operatorname{trn} \mathrm{F}$ (Taberlet et al., 1991; Demesure et al., 1995). Amplifications were performed in a DNA programmable thermal cycler (PTC-100, MJ Research, Watertown, MA, USA): initial denaturation at $94{ }^{\circ} \mathrm{C}$ for $3 \mathrm{~min}$ followed by 42 cycles of $1 \mathrm{~min}$ at $94{ }^{\circ} \mathrm{C}, 1 \mathrm{~min}$ annealing at 52 and $50{ }^{\circ} \mathrm{C}$, respectively for $\operatorname{trn} \mathrm{V}-\operatorname{trn} \mathrm{M}$ and $\operatorname{trn} \mathrm{F}-\operatorname{trn} \mathrm{L}, 90 \mathrm{~s}$ at $72{ }^{\circ} \mathrm{C}$, and a subsequent 10-min final extension at $72{ }^{\circ} \mathrm{C}$. The PCR mixture $(25 \mu \mathrm{L})$ contained $500 \mathrm{~mm} \mathrm{KCl}, 15 \mathrm{~mm} \mathrm{MgCl}_{2}, 0.01 \%$ gelatin, $100 \mathrm{~mm}$ Tris- $\mathrm{HCl}$ (pH 8.3), 1 mм dNTPs, $2 \mu \mathrm{m}$ Primer, 20 ng template DNA, $1 \mu \mathrm{g}$ RNase and $0.5 \mathrm{U}$ Taq polymerase (Amersham Biosciences). The PCR products were purified using a QiaGen purification kit and then sequenced in both directions using a Taq Dye Dideoxy Terminator Cycle Sequencing Kit (Applied Biosystems, Foster City, CA, USA) and Model ABI373A automated sequencer (Applied Biosystems). For sequencing we used the same primers as those used for amplification. All sequence polymorphisms were visually rechecked from chromatograms. Repeated sequencing was performed for variation, which was identified as singletons. GenBank accession numbers for trnL-trnF and trnV-trnM were AY819940-AY819944 and AY819945-AY819950, respectively, in M. thunbergii.

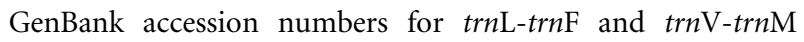
were AY819927-AY819932 and AY819933-AY819939, respectively, in M. kusanoi.

\section{Statistical analyses}

Multiple alignments of the sequences were obtained using ClustalW (Thompson et al., 1994) and subsequently adjusted manually. The number of haplotypes was measured within each sampling locality. Haplotype diversity (h), nucleotide diversity $(\pi)$ (Nei, 1987), Tajima's $D$ (Tajima, 1989), Fu and Li's $D^{\star}$ and Fu and Li's $F^{\star}$ test (Fu \& Li, 1993) for departure from neutrality on the total number of segregating sites were calculated using DNASP version 4.0 (Rozas et al., 2003). Each indel was recoded as a transitional substitution for the DNASP program, as indels are consistently and unambiguously alignable. Two measures of population differentiation $G_{\mathrm{ST}}$ and $N_{\mathrm{ST}}$ were analysed by the HAPSTEP program (Pons \& Petit, 1996). $G_{\mathrm{ST}}$ depends only on the frequencies of the haplotypes, but $N_{\mathrm{ST}}$ is influenced by both haplotype frequencies and the distances between haplotypes.

The network of chloroplastic haplotypes was reconstructed using the algorithm of statistical parsimony described by Templeton et al. (1992) and implemented in TCs version 1.06 (Clement et al., 2000). We used nested clade analysis (NCA) to infer the population history of M. thunbergii and M. kusanoi. The NCA nesting design was constructed by hand on the TCS haplotype network following the rules given by Templeton et al. (1987) and Templeton \& Sing (1993). The program GeoDis 2.2 (Posada et al., 2000) was used to calculate the various NCA distance measures and their statistical significance. All statistical analyses in GeoDis were performed using 1000 permutations. Results obtained from GeoDis were then interpreted using the revised inference key of Templeton (2004). The statistics calculated for all clades were: (i) clade distance $\left(D_{\mathrm{C}}\right)$, which measures the average distance of all clade members from the geographical centre of distribution; (ii) nested clade distance $\left(D_{\mathrm{N}}\right)$, which measures how widespread a particular clade is relative to the distribution of other clades in the same nesting group; and (iii) interior-tip distances $\left(\mathrm{I}-\mathrm{T} D_{\mathrm{C}}\right.$ and $\mathrm{I}-\mathrm{T} D_{\mathrm{N}}$ ). This interior vs. tip contrast of clades corresponds to younger clades (tip clade) relative to their ancestors' clades (interior clades), common vs. rare under expectations from neutral coalescent theory (Crandall \& Templeton, 1993). Testing for significantly small or large $D_{\mathrm{C}}$ or $D_{\mathrm{N}}$ distances in each nested clade is then used to reject the null distribution of no association between haplotype distributions and geography.

A Mantel test of the matrix of pairwise genetic distances against the matrix of pairwise geographical distances was performed by a simple Mantel test of zT: a software tool for simple and partial Mantel tests (Bonnet \& Van de Peer, 2002). The contribution of the populations to gene diversity and to allelic richness was calculated following Petit et al. (1998) using the computer software CONTRIB. The contribution of a population to the total diversity was calculated as the relative variation of the diversity on adding this population to the others. Similarly, the contribution to total diversity of a 
Table 1 Population code, sample size, localities, chloroplast haplotype diversity, and nucleotide diversity for Machilus thunbergii and Machilus kusanoi in Taiwan

\begin{tabular}{|c|c|c|c|c|c|}
\hline $\begin{array}{l}\text { Population } \\
\text { code }\end{array}$ & $\begin{array}{l}\text { Sample } \\
\text { size }\end{array}$ & $\begin{array}{l}\text { Locality } \\
\left({ }^{\circ} \mathrm{E} /{ }^{\circ} \mathrm{N}\right)\end{array}$ & $\begin{array}{l}\text { Haplotype } \\
\text { (no. of individuals) }\end{array}$ & $\begin{array}{l}\text { Haplotype } \\
\text { diversity }(h)\end{array}$ & $\begin{array}{l}\text { Nucleotide } \\
\text { diversity }(\pi) \times 10^{3}\end{array}$ \\
\hline \multicolumn{6}{|l|}{ M. thunbergii } \\
\hline Total & 110 & & & $0.165 \pm 0.049$ & $0.31 \pm 0.11$ \\
\hline 1 Qingrenhu & 4 & $121.42 / 25.09$ & MTA (4) & 0.000 & 0.00 \\
\hline 2 Yangmingshan & 6 & $121.51 / 25.18$ & MTA (6) & 0.000 & 0.00 \\
\hline 3 Pinglin & 4 & $121.70 / 24.92$ & MTA (4) & 0.000 & 0.00 \\
\hline 4 Sanxia & 7 & $121.42 / 24.56$ & MTA (7) & 0.000 & 0.00 \\
\hline 5 Zudong & 4 & $121.05 / 24.44$ & MTA (3), MTH (1) & $0.500 \pm 0.265$ & $0.97 \pm 0.52$ \\
\hline 6 Shiouhluan & 3 & $121.16 / 24.37$ & MTA (1), MTG (1), MTI (1) & $1.000 \pm 0.272$ & $1.94 \pm 0.68$ \\
\hline 7 Tashueshan & 5 & $121.36 / 24.15$ & MTA (2), MTB (2), MTD (1) & $0.800 \pm 0.164$ & $1.75 \pm 0.69$ \\
\hline 8 Beidongyeinshan & 3 & $121.21 / 24.49$ & MTA $(3)$ & 0.000 & 0.00 \\
\hline 9 JiYuetan & 5 & $120.54 / 23.52$ & MTA (4), MTF (1) & $0.400 \pm 0.237$ & $0.39 \pm 0.23$ \\
\hline 10 Tadongshan & 3 & $120.41 / 23.30$ & MTA (3) & 0.000 & 0.00 \\
\hline 11 Duona & 4 & $120.43 / 22.54$ & MTA (4) & 0.000 & 0.00 \\
\hline 12 Tahanshan & 4 & $120.40 / 22.24$ & MTA (4) & 0.000 & 0.00 \\
\hline 13 Tawushan & 4 & $120.54 / 22.21$ & MTA (4) & 0.000 & 0.00 \\
\hline 14 Taimali & 4 & $121.01 / 22.36$ & MTA $(4)$ & 0.000 & 0.00 \\
\hline 15 Jinping & 4 & $121.10 / 23.07$ & MTA (3), MTE (1) & $0.500 \pm 0.265$ & $0.97 \pm 0.52$ \\
\hline 16 Yakou & 4 & $120.58 / 23.15$ & MTA $(4)$ & 0.000 & 0.00 \\
\hline 17 Zhongping & 4 & $121.19 / 23.24$ & MTA (4) & 0.000 & 0.00 \\
\hline 18 Rueishuei & 4 & $121.42 / 23.50$ & MTA $(4)$ & 0.000 & 0.00 \\
\hline 19 Liyutan & 4 & $121.30 / 23.56$ & MTA (3), MTC (1) & $0.500 \pm 0.265$ & $1.94 \pm 1.03$ \\
\hline 20 Herpin & 4 & $121.42 / 24.18$ & MTA (4) & 0.000 & 0.00 \\
\hline 21 Wushibi & 5 & $121.49 / 24.28$ & MTA (5) & 0.000 & 0.00 \\
\hline 22 Lupi & 4 & $121.43 / 24.28$ & MTA (4) & 0.000 & 0.00 \\
\hline 23 Chilanshan & 4 & $121.29 / 24.35$ & MTA (4) & 0.000 & 0.00 \\
\hline 24 Fushan & 4 & $121.37 / 24.39$ & MTA (4) & 0.000 & 0.00 \\
\hline 25 Gueishan & 4 & $121.57 / 24.51$ & MTA (4) & 0.000 & 0.00 \\
\hline \multicolumn{6}{|l|}{ M. kusanoi } \\
\hline Total & 106 & & & $0.159 \pm 0.048$ & $0.51 \pm 0.22$ \\
\hline 1 Wulai & 10 & $121.34 / 24.51$ & mka (9), mkb (1) & $0.200 \pm 0.154$ & $0.19 \pm 0.15$ \\
\hline 2 Fushan & 10 & $121.35 / 24.46$ & mka $(10)$ & 0.000 & 0.00 \\
\hline 3 Zudong & 4 & $121.04 / 24.44$ & mka (4) & 0.000 & 0.00 \\
\hline 4 Miaoli & 8 & $120.45 / 24.22$ & mka $(8)$ & 0.000 & 0.00 \\
\hline 5 Herpin & 7 & $121.44 / 24.18$ & mka (6), mkj (1) & $0.286 \pm 0.196$ & $2.48 \pm 1.70$ \\
\hline 6 Lienhuachih & 5 & $120.53 / 23.55$ & mka (5) & 0.000 & 0.00 \\
\hline 7 Liyutan & 5 & $121.30 / 23.56$ & mka (4), mkd (1) & $0.400 \pm 0.237$ & $0.39 \pm 0.23$ \\
\hline 8 Fanlu & 5 & $120.33 / 23.27$ & mka (5) & 0.000 & 0.00 \\
\hline 9 Zhongpin & 6 & $121.18 / 23.23$ & mka (6) & 0.000 & 0.00 \\
\hline 10 Anton & 6 & $121.19 / 23.17$ & mka $(6)$ & 0.000 & 0.00 \\
\hline 11 Jiashein & 8 & $120.35 / 23.04$ & mka (7), mkh (1) & $0.250 \pm 0.180$ & $0.24 \pm 0.17$ \\
\hline 12 Dongher & 9 & $121.18 / 22.58$ & mka (8), mkg (1) & $0.222 \pm 0.166$ & $0.21 \pm 0.16$ \\
\hline 13 Honyeh & 5 & $121.04 / 22.54$ & mka (5) & 0.000 & 0.00 \\
\hline 14 Laiyi & 9 & $120.38 / 22.31$ & mka $(9)$ & 0.000 & 0.00 \\
\hline 15 Soukar & 5 & $120.50 / 22.14$ & mka (3), mkc (1), mki (1) & $0.400 \pm 0.237$ & $1.15 \pm 0.69$ \\
\hline 16 Kending & 4 & $120.48 / 21.58$ & mka (2), mke (1), mkf (1) & $0.833 \pm 0.222$ & $4.50 \pm 1.23$ \\
\hline
\end{tabular}

population due to its own diversity and to its divergence was calculated. The contribution of a population to the total allelic richness was calculated by following the rarefaction method of Hurlbert (1971). The contribution of this population due to its own diversity and to its divergence was obtained from the partitioning of total allelic richness in similar ways to the contribution to total diversity.
Conventional $F_{\mathrm{ST}}$ (Wright, 1931), based on cpDNA sequences for population subdivision, was estimated using Analysis of Molecular Variance (AMOVA) implemented in the ARLEQUIN program (Schneider et al., 2000). In addition, the level of divergence for each population from the remaining populations was calculated as the mean value of pairwise $F_{\mathrm{ST}}$ for each population against the rest of the populations. 


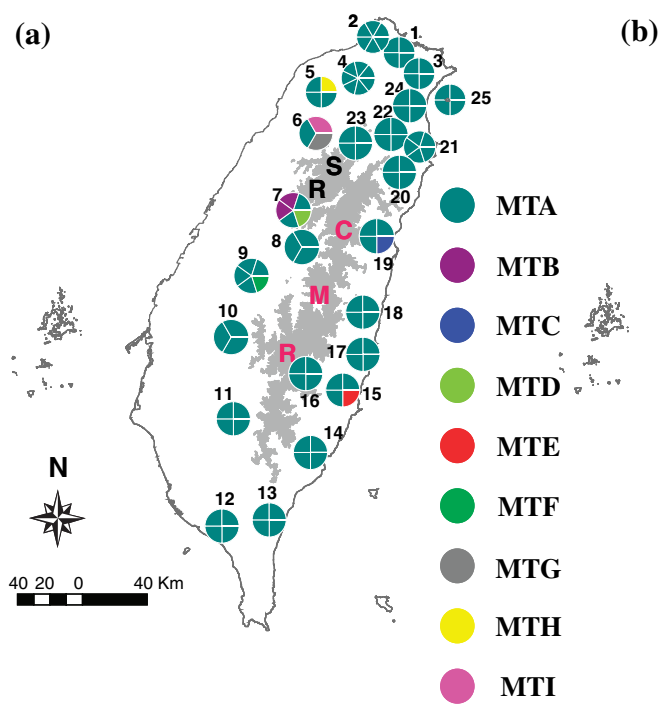

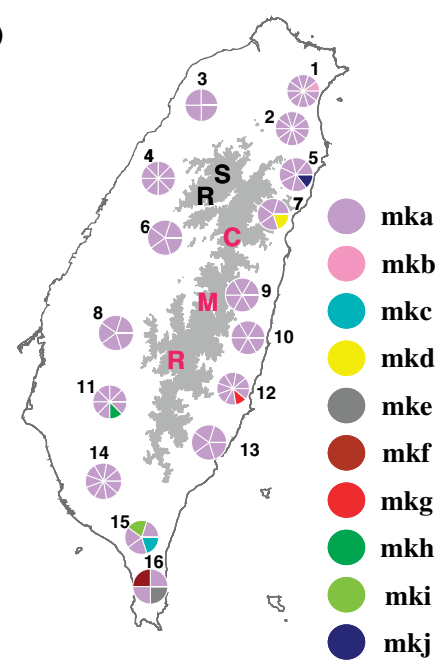

Figure 1 Map of Taiwan showing sampling sites and haplotype designations of (a) Machilus thunbergii; (b) Machilus kusanoi. The different number of pies in the haplotype circle for each population indicates the number of samples examined for each population. The shaded area indicates the Shueshan Range and Central Mountain Ridge in Taiwan.

\section{RESULTS}

\section{cpDNA variation and haplotype relationships}

Both $M$. thunbergii and M. kusanoi displayed very low levels of cpDNA nucleotide diversity (Table 1). Of the aligned 1031-bp sequences in $M$. thunbergii, 14 varied among nine haplotypes (indels at bases 109 and 110 were treated as one insertiondeletion event) (Table 2). In M. kusanoi, of the aligned $1041 \mathrm{bp}, 20$ polymorphic sites were observed among 10 haplotypes. Only one informative character was found at polymorphic site 162 in the $t r n \mathrm{~V}$-trn $\mathrm{M}$ region in M. thunbergii. In contrast, three (polymorphic sites at 13, 57 and 69) in trn V-trn $\mathrm{M}$ and two (polymorphic sites at 730 and 758) in $\operatorname{trn} \mathrm{L}-\operatorname{trn} \mathrm{F}$ were found in M. kusanoi. Haplotype MTA in $M$. thunbergii was fixed in 19 out of 25 populations investigated, with a haplotype frequency of 0.918 (Fig. 1). The haplotype frequency of rare alleles in $M$. thunbergii was either 0.009 or 0.018. In contrast, haplotype mka in M. kusanoi was fixed in nine out of the 16 populations examined, with a haplotype frequency of 0.915 . All the rare haplotypes in M. kusanoi had a haplotype frequency of 0.009 . Coalescent theory predicts that older (interior) haplotypes should be more common than derived (tip) haplotypes, and there is empirical evidence to support this idea (Crandall \& Templeton, 1993). Following the rules of Crandall \& Templeton (1993), tip haplotypes are defined as those connected to only one other haplotype, whereas interior haplotypes are connected to multiple haplotypes. The MTA haplotype in M. thunbergii is the most common and connected to multiple haplotypes (Figs $1 \& 2$ ). The most common haplotype is mka in M. kusanoi, which is connected to multiple haplotypes (Figs $1 \& 2$ ). Therefore haplotype MTA of $M$. thunbergii and mka of M. kusanoi are considered to be the ancestral haplotypes for M. thunbergii and M. kusanoi, respectively. Furthermore, only haplotype MTA was found in four individuals of $M$. thunbergii collected from Okinawa.
In $M$. thunbergii, the Shiouhluan and Liyutan populations had the highest level of nucleotide diversity, followed by Tashueshan. The highest cpDNA haplotype diversity was found in Shiouhluan, followed by Tashueshan. The M. kusanoi population in Kending had the highest level of nucleotide and haplotype diversity (Table 1). Moreover, the population group in the western CMR had higher haplotype and nucleotide diversities than the population group in the eastern CMR in M. thunbergii (Table 3). In contrast, the M. kusanoi population group in the eastern CMR had higher haplotype and nucleotide diversities than the population group in the western CMR.

\section{Neutral evolution, haplotype structure and population history inferred from NCA}

A neutrality test revealed a significant departure from equilibrium for all $25 \mathrm{M}$. thunbergii populations examined (Table 3). The neutrality test was also carried out on the population groups in the western and eastern CMR. The western group and the total populations had similar results, which showed a significant departure from equilibrium. A significant departure from equilibrium was also found for the total populations and for the eastern group of $M$. kusanoi populations examined (Table 3).

In $M$. thunbergii the $N_{\mathrm{ST}}$ estimate $\left(N_{\mathrm{ST}}=0.025\right)$ was smaller than the $G_{\mathrm{ST}}$ estimate $\left(G_{\mathrm{ST}}=0.161\right)$ in 25 populations, and the difference was significant (Table 3 ). This result indicated that haplotypes within populations had distant genetic relationships. A similar result was found for the populations west of the CMR. Mutation-drift equilibrium was found for the populations east of the CMR, with $N_{\mathrm{ST}}-G_{\mathrm{ST}}$ approaching zero. In $M$. kusanoi, the $N_{\mathrm{ST}}$ estimate $\left(N_{\mathrm{ST}}=0.111\right)$ was higher than the $G_{\mathrm{ST}}$ estimate $\left(G_{\mathrm{ST}}=0.044\right)$ in 16 populations, and the difference was significant (Table 3). Mutation-drift equilibrium was found for the populations west of the CMR in this species. It is also clear that, in M. kusanoi, the eastern 


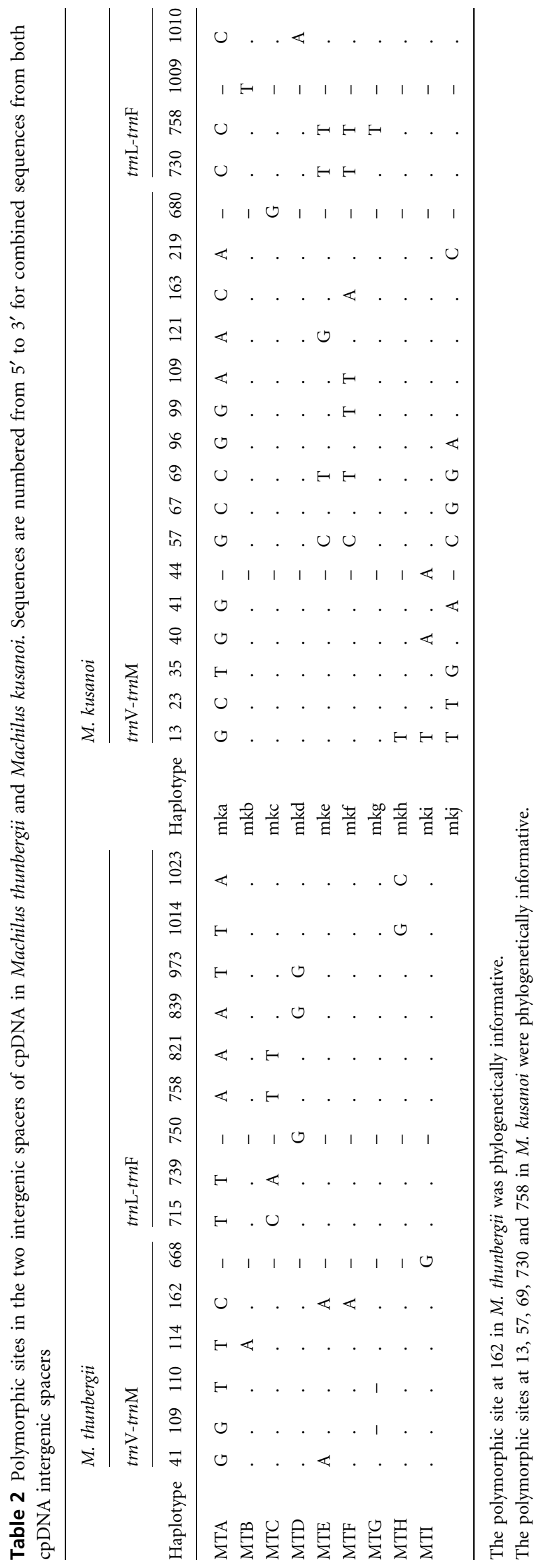

(a)

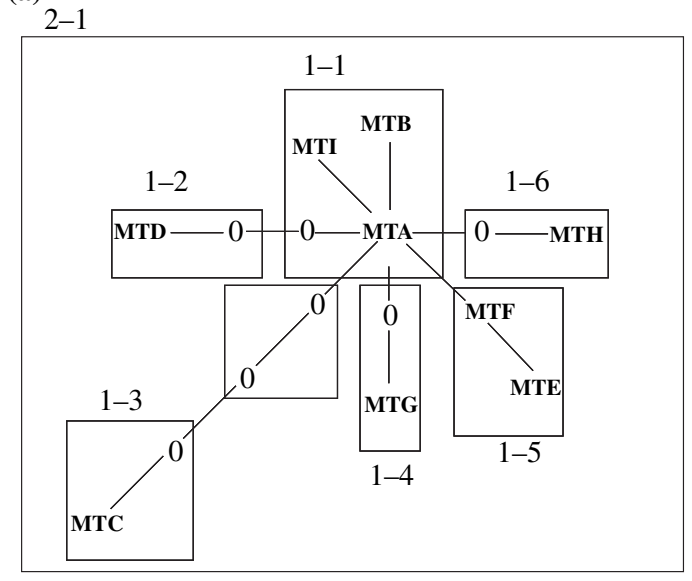

M.thunbergii

(b)

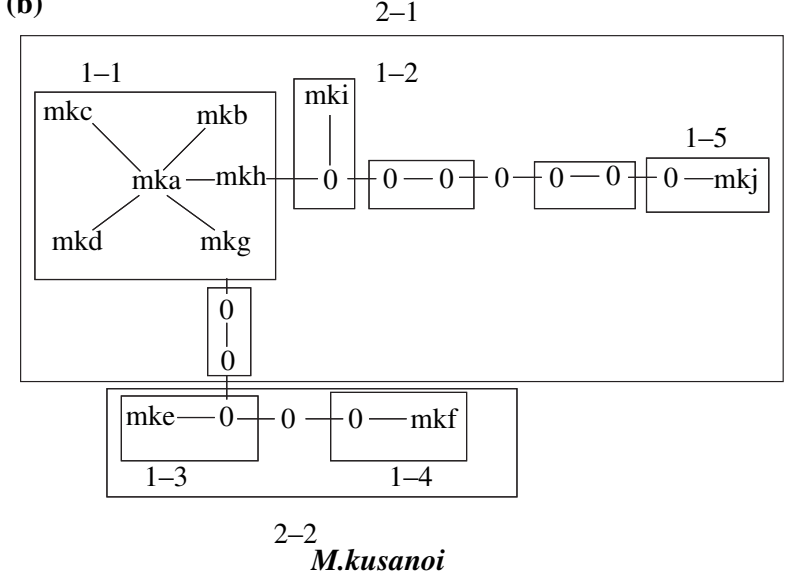

Figure 2 Nested cladogram of Machilus thunbergii (a) and Machilus kusanoi (b) haplotypes. Each line connecting one haplotype to another indicates one mutational step. Haplotype designations correspond to those in Table 1 and Fig. 1. Unsampled haplotypes are represented by 0 . Hierarchical nesting design is specified by boxes and numbered clade designations.

group of populations showed significant non-random distribution of haplotypes when the ordered and unordered alleles are compared with estimated population differentiation that corresponds to the higher genetic similarity found within populations. Both these two closely related species had low levels of population differentiation.

Nested clade analysis showed a marginal relationship between genetic and geographical distribution in M. thunbergii. However, a significant relationship between genetic and geographical distribution was observed in $M$. kusanoi (Table 4). In M. thunbergii, restricted gene flow with isolation-by-distance was inferred between the populations on the Sheushan Range (clade 1-1), with an inconclusive result for clade 1-5 (Fig. 2a). Although no conclusive result was inferred for the haplotypes in clade $1-1$ and one-step clade 2-1, contiguous range expansion was inferred from the NCA analysis of the two-step clade in M. kusanoi (Fig. 2b). 
Table 3 Neutrality tests for 25 Machilus thunbergii and 16 Machilus kusanoi populations in Taiwan based on chloroplast intergenic spacers of $\operatorname{trn} \mathrm{V}-\operatorname{trn} \mathrm{M}$ and $\operatorname{trn} \mathrm{L}-\operatorname{trn} \mathrm{F}$

\begin{tabular}{|c|c|c|c|}
\hline $\begin{array}{l}\text { Diversity parameter } \\
\text { and test statistic }\end{array}$ & Total & $\begin{array}{l}\text { West of } \\
\text { CMR } \dagger\end{array}$ & $\begin{array}{l}\text { East of } \\
\text { CMR } \$\end{array}$ \\
\hline \multicolumn{4}{|l|}{ M. thunbergii } \\
\hline$h$ & $0.165 \pm 0.049$ & $0.272 \pm 0.084$ & $0.070 \pm 0.046$ \\
\hline$\pi \times 10^{3}$ & $0.31 \pm 0.11$ & $2.19 \pm 0.90$ & $0.20 \pm 0.14$ \\
\hline$G_{\mathrm{ST}(\mathrm{SE})}$ & $0.161(\mathrm{NC})$ & $0.164(\mathrm{NC})$ & $0.01(\mathrm{NC})$ \\
\hline$N_{\mathrm{ST}(\mathrm{SE})}$ & $0.025(\mathrm{NC})$ & $0.037(\mathrm{NC})$ & 0.000 \\
\hline$N_{\mathrm{ST}}-G_{\mathrm{ST}}$ & -0.136 & -0.127 & -0.01 \\
\hline Tajima's $D$ & $-2.440^{\star *}$ & $-2.297^{\star \star}$ & $-2.079^{* *}$ \\
\hline $\mathrm{Fu}$ and Li's $D^{*}$ & $-5.527^{\star *}$ & $-4.073^{\star \star}$ & $-4.111^{* *}$ \\
\hline $\mathrm{Fu}$ and Li's $F^{\star}$ & $-5.245^{\star *}$ & $-4.114^{* *}$ & $-4.065^{\star *}$ \\
\hline \multicolumn{4}{|l|}{ M. kusanoi } \\
\hline$h$ & $0.159 \pm 0.048$ & $0.079 \pm 0.052$ & $0.19500 \pm 0.069$ \\
\hline$\pi \times 10^{3}$ & $0.51 \pm 0.22$ & $0.08 \pm 0.05$ & $0.84 \pm 0.38$ \\
\hline$G_{\mathrm{ST}(\mathrm{SE})}$ & $0.044(\mathrm{NC})$ & $-0.009(\mathrm{NC})$ & $0.039(\mathrm{NC})$ \\
\hline$N_{\mathrm{ST}(\mathrm{SE})}$ & $0.111(\mathrm{NC})$ & $0.000(\mathrm{NC})$ & $0.116(\mathrm{NC})$ \\
\hline$N_{\mathrm{ST}}-G_{\mathrm{ST}}$ & 0.067 & 0.009 & 0.077 \\
\hline Tajima's $D$ & $-2.467^{\star \star}$ & -1.464 & $-2.379^{* *}$ \\
\hline Fu and Li's $D^{*}$ & $-4.615^{\star \star}$ & $-2.533^{\star}$ & $-3.390^{\star}$ \\
\hline $\mathrm{Fu}$ and Li's $F^{\star}$ & $-4.547^{\star \star}$ & $-2.575^{\star}$ & $-3.604^{* *}$ \\
\hline
\end{tabular}

$\dagger$ Includes populations 1, 2, 3, 4, 5, 6, 7, 8, 9,10 and 11 in M. thunbergii; populations $1,3,4,6,8,11$ and 14 in M. kusanoi.

†Includes populations 12, 13, 14, 15, 16, 17, 18, 19, 20, 21, 22, 23, 24 and 25 in M. thunbergii; populations 2, 5, 7, 9, 10, 12, 13, 15 and 16 in M. kusanoi.

${ }^{\star} P<0.05 ;{ }^{* *} P<0.02$.

\section{Source populations of recolonization}

A Mantel test of the matrix of pairwise genetic distance against the matrix of pairwise geographical distance resulted in a negative relationship between these two matrices $(r=-0.1775$, $P=0.0035, \quad 10,000$ permutations, one-tailed test) for M. thunbergii. Similarly, a Mantel test of the genetic distance matrix against the matrix of geographical distances also resulted in a negative relationship for $M$. kusanoi $(r=-0.1383$, $P=0.0590,10,000$ permutations, one-tailed test). However, when the eastern group of M. kusanoi populations was analysed, the genetic distances increased significantly with geographical distance $(r=0.4108, P=0.027,10,000$ permutations, onetailed test). Nevertheless, if recolonization was derived from a small number of source populations through stepping-stone colonization, we might expect a pattern in which the most important source populations showed a stronger contribution to the total diversity and allelic richness. Therefore the contributions of each population to the total diversity and allelic richness were analysed (Petit et al., 1998). Results for M. thunbergii showed that the populations in Shiouhluan and Tashueshan contributed most to the divergence and to the diversity components of total diversity, due to their high haplotype diversity (Fig. 3a). The M. kusanoi populations in Soukar and Kending contributed most to the divergence and diversity components of total diversity (Fig. 3b). The two populations that contributed most to the divergence and diversity components of the total diversity in respective species also played an important role in the contribution of allelic richness. These results suggest that source populations do exist within local geographical areas.

Furthermore, the source population for recolonization from the centre of diversity can be further inferred from the mean $F_{\mathrm{ST}}$ for individual populations in comparison with the remaining populations. The mean $F_{\mathrm{ST}}$ results showed that the most genetically distinct populations were the area covering Shiouhluan and Tashueshan for M. thunbergii, and the area covering Kending for M. kusanoi (Fig. 4).

\section{DISCUSSION}

\section{Genetic diversity and population differentiation}

The low levels of cpDNA nucleotide diversity in $M$. thunbergii and M. kusanoi were consistent with other widespread tree species in Taiwan (Huang et al., 2002; Hwang et al., 2003;

Table 4 Inference of historical processes shaping the geographical distribution of Machilus thunbergii and Machilus kusanoi cpDNA haplotypes. Inferences are made from relative values of dispersion distance $\left(D_{\mathrm{C}}\right)$ and displacement distance $\left(D_{\mathrm{N}}\right)$ of tip and interior haplotype/clade (Fig. 2)

\begin{tabular}{|c|c|c|c|c|c|c|}
\hline Nested clade & $\begin{array}{l}\text { Permutational } \\
\chi^{2} \text { statistic }\end{array}$ & $P$ & $D_{\mathrm{C}}$ & $D_{\mathrm{N}}$ & $\begin{array}{l}\text { Inference } \\
\text { chain }\end{array}$ & Inferred pattern \\
\hline \multicolumn{7}{|l|}{ M. thunbergii } \\
\hline $1-1$ & 72.445 & $0.0490^{*}$ & $95.56^{\mathrm{L}}$ & $95.50^{\mathrm{L}}$ & $1-2-3-4-\mathrm{NO}$ & $\begin{array}{l}\text { Restricted gene flow with } \\
\text { isolation-by-distance }\end{array}$ \\
\hline $1-5$ & & & 37.50 & 77.69 & 1-19-20-2-11-17-NO & Inconclusive outcome \\
\hline Total cladogram & 136.880 & 0.0510 & 83.07 & $36.71^{\mathrm{L}}$ & $1-2-3-4-\mathrm{NO}$ & $\begin{array}{l}\text { Restricted gene flow with } \\
\text { isolation-by-distance }\end{array}$ \\
\hline \multicolumn{7}{|l|}{ M. kusanoi } \\
\hline $1-1$ & 75.752 & 0.4380 & 91.31 & 91.29 & 1-2-11-17-NO & Inconclusive outcome \\
\hline $2-1$ & 38.432 & 0.1230 & $92.10^{\mathrm{S}}$ & $92.09^{\mathrm{S}}$ & $1-2-11-17-\mathrm{NO}$ & Inconclusive outcome \\
\hline Total cladogram & 51.481 & $0.0020^{*}$ & 92.74 & $-102.99^{S}$ & $1-2-11-12-\mathrm{NO}$ & Contiguous range expansion \\
\hline
\end{tabular}

$\mathrm{S}$ and $\mathrm{L}=D_{\mathrm{C}}$ or $D_{\mathrm{N}}$ values that are significantly smaller $(\mathrm{S}) /$ larger $(\mathrm{L})$ than expected at the $5 \%$ level based on 1000 permutations. ${ }^{\star} P<0.05$. 
(a)
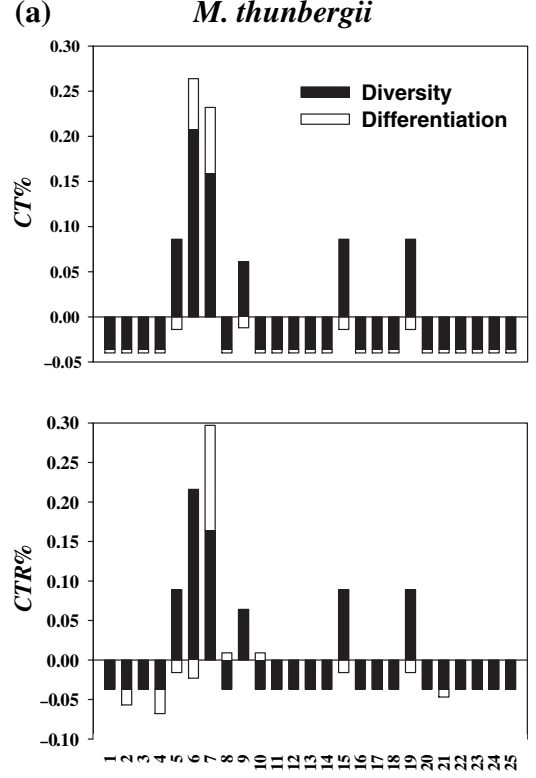

Populations (b)
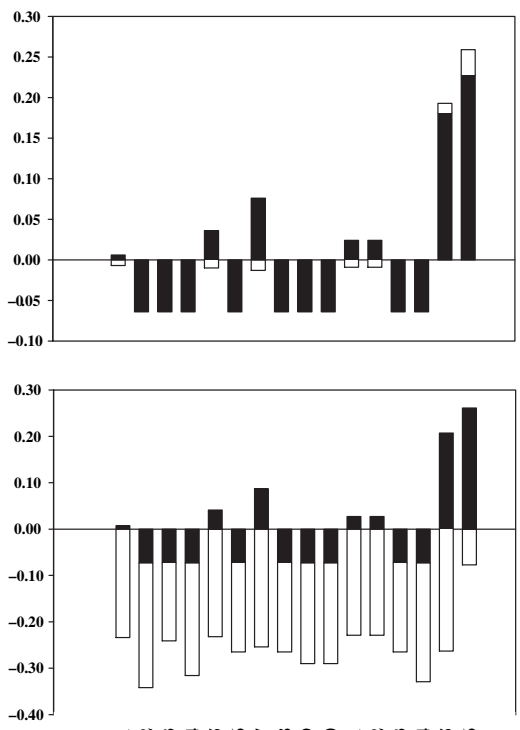

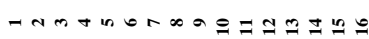

Populations

Huang et al., 2004). For the two closely related species investigated here, a contrasting level of cpDNA nucleotide diversity was found, as the same cpDNA intergenic spacer harboured different levels of nucleotide diversity in these two closely related species. It is also apparent that the island endemic M. kusanoi had a greater number of variable/ informative molecular characters than its mainland counterpart M. thunbergii. This result is generally consistent with the more rapid evolutionary rate found in the island endemic species (Bromham \& Woolfit, 2004). The higher level of cpDNA nucleotide diversity found in M. kusanoi compared with M. thunbergii is probably related to the faster rate of recolonization after cold periods in the Pleistocene.

Rates and patterns of dispersal and migration between populations will affect the genetic structure of species: the higher the dispersal ability, the lower the population structuring (Clegg et al., 2002). Local adaptation and genetic drift may cause considerable phenotypic variation in widespread species (Joshi et al., 2001). Populations along a colonization line following a glacial bottleneck can have a very low population differentiation $\left(F_{\mathrm{ST}}=0.005\right)$, as found by maternal inherited mitochondrial DNA markers in Pinus flexilis (Latta \& Mitton, 1997). This result was tested further, and suggested by the simulation of a onedimensional colonization model for angiosperm tree species (Austerlitz et al., 2000). The levels of genetic structure are unusually low in both species of Machilus; investigations of maternally inherited cpDNA in most angiosperms resulted in high levels of population differentiation (Petit et al., 2005). We think that the low level of population differentiation found in Machilus may be related to the evolutionary history of this species, in which multiple relict refugia mainly hosted the ancestral haplotype. Little differentiation in the cpDNA of all populations investigated was equivocal with respect to the 
multiple refugia hypothesis. Similar results have been documented by Soltis et al. (1997). Furthermore, if all haplotypes of M. kusanoi within a nesting level originated from a major source population, then relatively little genetic differentiation between haplotypes would be predicted.

\section{Geographical patterns of genetic variation}

It is typically reported that Pleistocene glaciation reduced species genetic variation in glaciated regions compared with regions that remained mostly ice-free. Although Taiwan was not glaciated, except for the high mountain peaks, the annual average temperature was $8-11^{\circ} \mathrm{C}$ cooler (Tsukada, 1966), and a remarkably high proportion of grasses (Poaceae) indicates a dry environment in central Taiwan 20,000 years ago (Liew \& Chung, 2001). This dry environment caused the deciduous broadleaved forests dominated by Alnus to replace the preexisting evergreen broadleaved Machilus-Castanopsis forests in low, hilly areas (Liew \& Chung, 2001). It is possible that most contemporary populations of $M$. thunbergii and M. kusanoi in Taiwan are descended from remnant populations that survived in some relict refugia during cold periods of the Pleistocene, and thus the ancestral haplotypes (MTA and mka) in both species are fixed in the majority of populations examined.

The significantly larger $G_{\mathrm{ST}}$ compared with $N_{\mathrm{ST}}$ indicated a random distribution of haplotypes in $M$. thunbergii. Multiple relict refugia existing during a period of cold climate, that hosted mainly the ancestral haplotype, would probably cause the shallow sequence divergence between haplotypes in $M$. thunbergii, which represents only an ancestral relationship. It is likely that the ancestral haplotype MTA became fixed by genetic drift in different relict refugial populations and therefore existed before the Holocene. It is interesting that cpDNA haplotypes showed a deeper sequence divergence in the haplotype network of M. kusanoi. Shen (1997) proposed that the flora of Taiwan could be divided into a southern Taiwan floristic superdistrict and a north-western Taiwan floristic superdistrict. The boundary between these two floristic superdistricts begins at Tahanshan (south Taiwan), proceeds northward along the eastern side of the CMR, and ends at Chingshuishan, close to the population at Herpin. The southern Taiwan floristic-superdistrict hypothesis, together with the non-random distribution of haplotypes (larger $N_{\mathrm{ST}}$ compared with $G_{\mathrm{ST}}$ ) in M. kusanoi, allows at least two putative lineages to be inferred, although haplotypes in the lineages differ by several missing steps. The haplotypes mkh, mki and mkj formed one lineage; a second lineage included haplotypes $\mathrm{mkg}$, mke and mkf (Fig. 2). Other rare haplotypes were probably derived randomly from ancestral haplotypes. Dominance of these two lineages in the eastern populations suggests the presence of a major source population at the southern tip of the island. It has been documented that interglacial periods became warmer and wetter compared with glacial periods, due to the intensification of summer monsoons (Huang et al., 1997). One possible explanation for the longer branches exhibited by the southern haplotypes in M. kusanoi is that the trend of temperature rise after cold climate periods of the Pleistocene occurred from south to north, facilitating the growth and expansion of M. kusanoi at lower altitudes. In contrast, suitable habitats for $M$. thunbergii were at relatively higher altitudes, thereby constraining the recolonization process.

\section{Phylogeographical patterns and local source populations of recolonization}

Despite considerable interest in the effects of past climate change on plant migration, relatively little is known about the historical processes that have shaped present-day genetic diversity in regions such as Taiwan, which were mostly unglaciated. The geographical structuring of cpDNA haplotypes provided insights into the post-glacial history of two closely related species, M. thunbergii and M. kusanoi, within Taiwan.

Significant negative values obtained in a neutrality test for $M$. thunbergii may have been contributed mainly by the haplotypes collected from the area covering Tashueshan and Shiouhluan. However, this may not reflect a range-expansion event as a whole. Moreover, negative values of neutrality test statistics reflect an excess of rare haplotypes, which is consistent with either positive selection or an increase in population size. As the DNA fragments under investigation are not subjected to selective forces, it is possible that an increase in population size can be inferred. However, mismatch distribution analysis (data not shown) does not agree with the inference of an increase in population size. Nevertheless, the power is conservative in deciphering population history because mismatch distributions use little information accumulated in the data (Felsenstein, 1992; Ramos-Onsins \& Rozas, 2002). Regional expansion in the area covering clade 1-1 in M. thunbergii did occur (Fig. 2a). This result can be explained by a short time to polymorphism accumulation recovered from an evolutionarily recent population bottleneck affecting $M$. thunbergii populations.

The haplotypes within clade 1-1 (haplotypes MTA, MTB and MTI; Fig. 2a) of M. thunbergii are limited primarily to the neighbouring populations at Tashueshan and Shiouhluan. This suggests a restricted gene flow with isolation-by-distance in this area in the north-central area west of the CMR. Indeed, NCA inferred a restricted gene flow event with isolationby-distance in clade 1-1 (Table 4). However, the occurrence of ancestral haplotype MTA in all the populations investigated suggests that multiple refugia existed throughout the island, although most probably had small population sizes. Haplotypes MTF and MTE in clade 1-5 occur in two separate populations to the west and east of the CMR. This pattern allows no conclusion from NCA analysis, which further supports the multiple refugia hypothesis for M. thunbergii. Furthermore, populations in Shiouhluan and Tashueshan had the highest haplotype and nucleotide diversities, indicating that the area is probably the source of restricted gene flow, as revealed by the NCA analysis at a local scale. This is further supported by analysis of the contribution of total diversity by 
divergence component (Fig. 3a). Previously, geographical regions in the north-central area, which is to the west of the CMR in Taiwan, have been proposed as the major diversity centre for several forest species based on allozyme variation (Lin, 2001). This north-central diversity centre in the western CMR was further revealed by amplified fragment length polymorphism (AFLP) analysis (Chung et al., 2004). Although the contribution from the divergence component to the total diversity was not found for the eastern populations, Jinping and Liyutan contributed some allelic richness.

Although neutrality test statistics obtained were significantly negative, the 16 sampled populations of M. kusanoi show a mismatching distribution pattern that does not fit the rangeexpansion model (data not shown). However, as mentioned above, that mismatch distribution analysis has low power in detecting range expansion. In contrast, the Mantel test showed significant migration patterns for the eastern group of $M$. kusanoi populations, and the population history of contiguous range expansion was obtained from the NCA analysis (Table 4). NCA inference of contiguous range expansion or long-distance colonization is thought to be conservative, and not prone to false positives (Templeton, 2002). In M. kusanoi, the conclusion of a contiguous range expansion by NCA analysis is consistent with the analysis of isolation-by-distance (Mantel test) for the eastern group of populations, as the Mantel test found a significant correlation between geographical and genetic distances for the eastern group of populations. The haplotypes mka, $\mathrm{mkb}, \mathrm{mkd}, \mathrm{mkg}, \mathrm{mkh}$ and $\mathrm{mki}$ are nested in clade $1-1$, and are distributed along the eastern side of the CMR. Total diversity contribution analysis suggests that Kending contributed most to the divergence component, which supports the idea of migration along the eastern side of the CMR for M. kusanoi.

\section{Comparison with Cyclobalanopsis glauca}

Cyclobalanopsis glauca is also a widely distributed species in East Asia, and is the most commonly occurring plant among the 50 native species of the family Fagaceae in Taiwan (Huang et al., 2002). Cyclobalanopsis glauca is found from sea level up to $1700 \mathrm{~m}$, and is one of the dominant species in the MachilusCastanopsis forests (Hsieh et al., 1994). It is interesting that the distributional range of $C$. glauca covers the distributional range of $M$. thunbergii and M. kusanoi. Three populations of C. glauca, Yangmingshan (northern Taiwan), Wushe (central Taiwan close to the Tashueshan population of M. thunbergii), and Tahanshan (southern Taiwan close to the Kending population of M. kusanoi), showed higher haplotype diversity than other populations. Therefore the diversity centres of C. glauca corresponded to that of $M$. thunbergii in central Taiwan and to that of M. kusanoi in southern Taiwan, although they did not completely match geographically.

\section{Conservation implications}

Understanding patterns of genetic variation within tree species is of fundamental importance for successful management in tree-conservation programmes. Without knowledge of possible adaptive differences among areas, assessment of biodiversity within and among populations is central to revealing information on the population evolution of the closely related species M. thunbergii and M. kusanoi. The information can then be used in identifying and prioritizing areas with comparatively high genetic diversity for monitoring, management and protection. Knowledge of population structure is important for ex situ and in situ conservation of natural populations (Williams \& Hamrick, 1996) by maintaining the total evolutionary potential and minimizing consanguinity. The present data on patterns of genetic diversity and phylogeographical structure suggest that the diversity centres, as well as some relict populations of both Machilus species, are worthy of conservation. The results of this investigation stress the importance of across-species range surveys to ensure a more representative sampling of the genetic diversity. Any ex situ conservation strategy should aim to include populations from both potential diversity centres, and from populations harbouring rare alleles for species that maintain mostly ancestral haplotypes.

\section{ACKNOWLEDGEMENTS}

This investigation was funded by the National Science Council (Grant Nos 91-2313-B-034-010 and 92-3114-B-034001), Executive Yuan, Taiwan. We would like to express our sincere gratitude to Shen-Ming Li (Taiwan Forestry Bureau), Ji-Shen Wu, Shu-Huei Wu and Wein-Chi Lin (Taiwan Forestry Research Institute), and Mrs Asataka Higa and Makoto Hirata, Okinawa Forestry Research Institute, Okinawa Prefecture (samples from Okinawa) for their help in the sample collection of several populations. Our gratitude also goes to Dr Mark Barnes, Department of Natural Resources, Chinese Culture University, who corrected the English for us.

\section{REFERENCES}

Austerlitz, F., Mariette, S., Machon, N., Gouyon, P.H. \& Godelle, B. (2000) Effects of colonization processes on genetic diversity: differences between annual plants and tree species. Genetics, 154, 1309-1321.

Avise, J.C. (2000) Phylogeography: the history and formation of species. Harvard University Press, Cambridge, MA.

Bonnet, E. \& Van de Peer, Y. (2002) zT: A software tool for simple and partial Mantel tests. Journal of Statistical Software, 7, 1-12.

Böse, M. (2004) Traces of glaciation in the high mountains of Taiwan. Quaternary glaciations - extent and chronology. Part III: Asia, Latin America, Africa, Australasia, Antarctica (ed. by J. Ehlers and P.L. Gibbard), pp. 347-352. Elsevier, Amsterdam.

Bromham, L. \& Woolfit, M. (2004) Explosive radiations and the reliability of molecular clocks: island endemic radiations as a test case. Systematic Biology, 53, 758-766. 
Chung, J.D., Lin, T.P., Tan, Y.C., Lin, M.Y. \& Hwang, S.Y. (2004) Genetic diversity and biogeography of Cunninghamia konishii (Cupressaceae), an island species in Taiwan: a comparison with Cunninghamia lanceolata, a mainland species in China. Molecular Phylogenetics and Evolution, 33, 791-801.

Clegg, S.M., Degnan, S.M., Kikkawa, J., Moritz, C., Estoup, A. \& Owens, I.P.F. (2002) Genetic consequences of sequential founder events by an island-colonizing bird. Proceedings of the National Academy of Sciences of the United States of America, 99, 8127-8132.

Clement, M., Posada, D. \& Crandall, K.A. (2000) тcs: A computer program to estimate gene genealogies. Molecular Ecology, 9, 1657-1659.

Crandall, K.A. \& Templeton, A.R. (1993) Empirical tests of some predictions from coalescent theory with applications to intraspecific phylogeny reconstruction. Genetics, 134, 959-969.

Demesure, B., Sodzi, N. \& Petit, R.J. (1995) A set of universal primers for amplification of polymorphic non-coding regions of mitochondrial and chloroplast DNA in plants. Molecular Ecology, 4, 129-131.

Doyle, J.J. \& Doyle, J.L. (1987) A rapid DNA isolation procedure for small quantities of fresh leaf material. Phytochemistry Bulletin, 19, 11-15.

Ennos, A.R. (1994) Estimating the relative rates of pollen and seed migration among plant populations. Heredity, 72, 250259.

Felsenstein, J. (1992) Estimating effective population size from samples of sequences: inefficiency of pairwise and segregating sites as compared to phylogenetic estimates. Genetical Research, 59, 139-147.

Fu, Y.X. \& Li, W.H. (1993) Statistical tests of neutrality of mutations. Genetics, 133, 693-709.

Hsieh, C.F., Shen, C.F. \& Yang, K.C. (1994) Introduction to the flora of Taiwan, 3: floristics, phytogeography, and vegetation. Flora of Taiwan, 2nd edn, Vol. 1 (ed. by Editorial Committee of the Flora of Taiwan), pp. 7-18. Editorial Committee of the Flora of Taiwan, Taipei.

Huang, C.Y., Liew, P.M., Zhao, M., Chang, T.C., Kuo, C.M., Chen, M.T., Wang, C.H. \& Zheng, L.F. (1997) Deep sea and lake records of the Southeast Asian paleomonsoons for the last 25 thousand years. Earth and Planetary Science Letters, 146, 59-72.

Huang, S.S.F., Hwang, S.Y. \& Lin, T.P. (2002) Spatial pattern of chloroplast DNA variation of Cyclobalanopsis glauca in Taiwan and East Asia. Molecular Ecology, 11, 23492358.

Huang, S.F., Hwang, S.Y., Wang, J.C. \& Lin, T.P. (2004) Phylogeography of Trochodendron aralioides (Trochodendraceae) in Taiwan and its adjacent areas. Journal of Biogeography, 31, 1251-1259.

Hurlbert, S.H. (1971) The nonconcept of species diversity: a critique and alternative parameters. Ecology, 52, 577-586.

Hwang, S.Y., Lin, H.W., Kuo, Y.S. \& Lin, T.P. (2001) RAPD variation in relation to population differentiation of Cha- maecyparis formosensis and Chamaecyparis taiwanensis. Botanical Bulletin of Academia Sinica, 42, 173-179.

Hwang, S.Y., Lin, T.P., Ma, C.S., Lin, C.L., Chung, J.D. \& Yang, J.C. (2003) Postglacial population growth of Cunninghamia konishii (Cupressaceae) inferred from phylogeographical and mismatch analysis of chloroplast DNA variation. Molecular Ecology, 12, 2689-2695.

Joshi, J., Schmid, B., Caldeira, M.C., Dimitrakopoulos, P.G., Good, J., Harris, R., Hector, A., Huss-Danell, K., Jumpponen, A., Minns, A., Mulder, C.P.H., Pereira, J.S., Prinz, A., Scherer-Lorenzen, M., Siamantziouras, A.S.D., Terry, A.C., Troumbis, A.Y. \& Lawton, J.H. (2001) Local adaptation enhances performance of common plant species. Ecology Letters, 4, 536-544.

Knowles, L.L. \& Maddison, W.P. (2002) Statistical phylogeography. Molecular Ecology, 10, 2623-2635.

Latta, R.G. \& Mitton, J.B. (1997) A comparison of population differentiation across four classes of gene markers in Limber Pine (Pinus flexilis James). Genetics, 146, 11531163.

Liew, P.M. \& Chung, N.J. (2001) Vertical migration of forests during the last glacial period in subtropical Taiwan. Western Pacific Earth Science, 1, 405-414.

Lin, T.P. (2001) Allozyme variations in the Michelia formosana (Kanehira) Masamune (Magnoliaceae), and the inference of a glacial refugium in Taiwan. Theoretical and Applied Genetics, 102, 450-457.

Liu, Y.C., Lu, F.Y. \& Ou, C.H. (1994) Trees of Taiwan. College of Agriculture, National ChungHsin University, Taiehung, Taiwan.

Nei, M. (1987) Molecular evolutionary genetics. Columbia University Press, New York.

Petit, R.J., El. Mousadik, A. \& Pons, O. (1998) Identifying populations for conservation on the basis of genetic markers. Conservation Biology, 12, 844-855.

Petit, R.J., Duminil, J., Fineschi, S., Salvini, D. \& Vendramin, G.G. (2005) Comparative organization of chloroplast, mitochondrial and nuclear diversity in plant populations. Molecular Ecology, 14, 689-701.

Pons, O. \& Petit, R.J. (1996) Measuring and testing genetic differentiation with ordered versus unordered alleles. Genetics, 144, 1237-1245.

Posada, D., Crandall, K.A. \& Templeton, A.R. (2000) GeoDis: a program for the cladistic nested analysis of the geographical distribution of genetic haplotypes. Molecular Ecology, 9, 487-488.

Ramos-Onsins, S.E. \& Rozas, J. (2002) Statistical properties of new neutrality tests against population growth. Molecular Biology and Evolution, 19, 2092-2100.

Rozas, J., Sànchez-DelBarrio, J.C., Messequer, X. \& Rozas, R. (2003) DNASP, DNA polymorphism analyses by the coalescent and other methods. Bioinformatics, 19, 2496-2497.

Schneider, S., Roessli, D. \& Excoffier, L. (2000) ARLEquin ver. 2.000: a software for population genetic data analysis. Genetics and Biometry Laboratory, University of Geneva, Geneva. 
Shen, C.F. (1997) The Biogeography of Taiwan: 2. Some preliminary thoughts and studies. Annual Report of Taiwan Museum, 40, 361-450 (in Chinese with English summary).

Soltis, D.E., Gitzendanner, M.A., Strenge, D.D. \& Soltis, P.A. (1997) Chloroplast DNA intraspecific phylogeography of plants from the Pacific Northwest of North America. Plant Systematics and Evolution, 206, 353-373.

$\mathrm{Su}, \mathrm{H}$.J. (1984) Studies on the climate and vegetation types of the natural forests in Taiwan: 1. Analysis in variation of climate factors. Quarterly Journal of Chinese Forestry, 17, 114 (in Chinese with English summary).

Taberlet, P., Gielly, L., Pautou, G. \& Bouvet, J. (1991) Universal primers for amplification of three non-coding regions of chloroplast DNA. Plant Molecular Biology, 17, 1105-1109.

Tajima, F. (1989) Statistical method for testing the neutral mutation hypothesis by DNA polymorphism. Genetics, 123, 585-595.

Templeton, A.R. (2002) Out of Africa again and again. Nature, 416, 45-51.

Templeton, A.R. (2004) Statistical phylogeography: methods of evaluating and minimizing inference errors. Molecular Ecology, 4, 789-809.

Templeton, A.R. \& Sing, C.F. (1993) A cladistic analysis of phenotypic associations with haplotypes inferred from restriction endonuclease mapping. IV. Nested analysis with cladogram uncertainty and recombination. Genetics, 134, 659-669.

Templeton, A.R., Boerwinkle, E. \& Sing, C.F. (1987) A cladistic analysis of phenotypic associations with haplotypes inferred from restriction endonuclease mapping. I. Basic theory and an analysis of alcohol dehydrogenase activity in Drosophila. Genetics, 117, 343-351.

Templeton, A.R., Crandall, K.A. \& Sing, C.F. (1992) A cladistic analysis of phenotypic associations with haplotypes inferred from restriction endonuclease mapping and DNA sequence data. III. Cladogram estimation. Genetics, 132, 619-633.

Thompson, J.D., Higgins, D.G. \& Gibon, T.J. (1994) ClusTALW: improving the sensitivity of progressive multiple sequence alignment through sequence weighting, positionspecific gap penalties and weight matrix choice. Nucleic Acids Research, 22, 4673-4680.
Tsukada, M. (1966) Late Pleistocene vegetation and climate in Taiwan (Formosa). Proceedings of the National Academy of Sciences of the United States of America, 55, 543-548.

Williams, C.G. \& Hamrick, J.L. (1996) Elite populations for conifer breeding and gene conservation. Canadian Journal of Forest Research, 26, 453-461.

Wright, S. (1931) Evolution in Mendelian populations. Genetics, 16, 97-159.

\section{BIOSKETCHES}

Su-Hwa Wu was a graduate student in the Graduate Institute of Biotechnology, Chinese Culture University. Her work mainly focuses on the phylogeography of Machilus thunbergii.

Ching-Yuan Hwang is a research assistant in the Graduate Institute of Biotechnology, Chinese Culture University under the supervision of Dr Shih-Ying Hwang. He conducts projects on the evolutionary genetics of endemic vascular plant species of Taiwan.

Tsan-Piao Lin is a professor in the Institute of Plant Biology, National Taiwan University. He is working on the population genetics and phylogeography of many native plant species in Taiwan.

Jeng-Der Chung is a research scientist in the Taiwan Forest Research Institute. He is working on breeding and conservation of endemic forest tree species in Taiwan.

Yu-Pin Cheng is a research scientist in the Taiwan Forest Research Institute. He is working on the phylogeography of endemic forest tree species in Taiwan.

Shih-Ying Hwang is a professor in the Graduate Institute of Biotechnology, Chinese Culture University. He is working on phylogeography and speciation of native vascular plants in Taiwan.

Editor: John Birks 